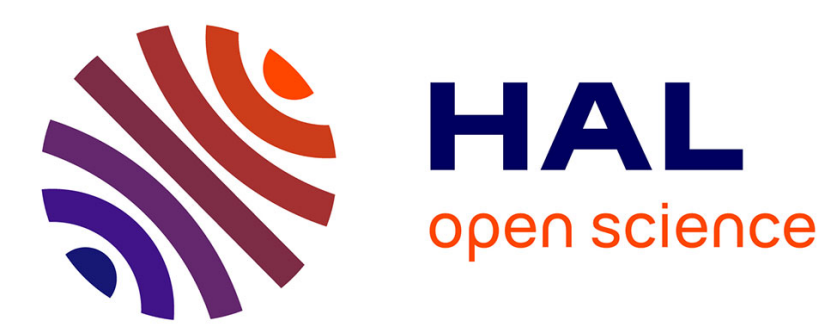

\title{
A Damage Model for the Dynamic Fragmentation of Brittle Solids
}

Christophe Denoual, François Hild

\section{To cite this version:}

Christophe Denoual, François Hild. A Damage Model for the Dynamic Fragmentation of Brittle Solids. Computer Methods in Applied Mechanics and Engineering, 2000, 183, pp.247-258. 10.1016/S00457825(99)00221-2 . hal-00013966

\section{HAL Id: hal-00013966 https://hal.science/hal-00013966}

Submitted on 17 Dec 2007

HAL is a multi-disciplinary open access archive for the deposit and dissemination of scientific research documents, whether they are published or not. The documents may come from teaching and research institutions in France or abroad, or from public or private research centers.
L'archive ouverte pluridisciplinaire HAL, est destinée au dépôt et à la diffusion de documents scientifiques de niveau recherche, publiés ou non, émanant des établissements d'enseignement et de recherche français ou étrangers, des laboratoires publics ou privés. 


\title{
A DAMAGE MODEL FOR \\ THE DYNAMIC FRAGMENTATION OF BRITTLE SOLIDS *
}

\author{
Christophe Denoual ${ }^{\mathrm{a}}$ and François Hild ${ }^{\mathrm{b}, 1}$ \\ ${ }^{a}$ DGA/CTA-Département Surfaces et Matériaux en Conditions Sévères \\ 16 bis avenue Prieur de la Côte d'Or, F-94114 Arcueil Cedex, France. \\ ${ }^{\mathrm{b}}$ LMT - Cachan \\ E.N.S. de Cachan / C.N.R.S. / Université Paris 6 \\ 61 avenue du Président Wilson, F-94235 Cachan Cedex, France.
}

\begin{abstract}
Impact produces high stress waves leading to the degradation of brittle materials such as ceramics. Based on a probabilistic approach, single and multiple fragmentation regimes are exhibited. The deterministic nature of the numerical simulation is discussed with respect to stress rate and volume. A damage model is proposed to account for dynamic loadings. Characteristic parameters are proposed and used to choose the mesh size. The mesh sensitivity is studied on a spalling configuration. Numerical predictions are compared with experimental data obtained on Edge-On Impact configurations.
\end{abstract}

\footnotetext{
* This work was funded by DGA-DRET-STRDT.

1 to whom correspondence should be addressed. Fax: +33 147402192 -
} 


\section{INTRODUCTION}

Bilayered armor with ceramics as front plate and steels as back face has been used for several years to improve the efficiency of light or medium armors [1]. The high hardness of ceramic materials favors projectile blunting and/or failure and spreads the kinetic energy on a large surface of the ductile back face. The weight of the armor is then reduced in comparison to an armor made of steel only. During the first micro-seconds of impact, high stress waves are produced and lead to a complete fragmentation of ceramics. Prior to penetration, stress waves can produce degradation in both compressive and tensile modes in different locations within the ceramic. Damage in compression is generated near the impact surface. Damage in tension is observed in a wide zone in the bulk of the ceramic and makes the complete perforation dependent on the way the ceramic fractures. The description of damage (in terms of location and kinetics, and in terms of anisotropic behavior due to cracking) is then one of the main mechanisms to identify for numerical simulations of impacts.

In Section 2, a probabilistic approach of the tensile fragmentation is derived. A first part briefly summarizes the information needed to describe single fragmentation. Multiple fragmentation is then analyzed as an extension of the single fragmentation regime by considering crack interactions. A scaling strategy is discussed when a constant stress rate is applied. Lastly, the transition between single and multiple fragmentation is studied. In particular, this transition can help in choosing the stress rates for which a discrete problem may be written on a continuum level. It can be noted that some computational modeling directly uses discrete descriptions of fracture as shown in [2] for instance

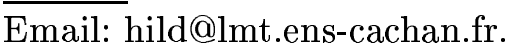


and models based upon Smoothed Particle Hydrodynamics [3,4] to simulate the behavior of brittle materials under shock loading [5].

To account for microcracking under dynamic loading conditions, the early models are based upon the analysis of cracked systems $[6,7]$ on a continuum level at which constitutive equations are written. The models developed by Taylor et al. [8] and Rajendran [9] use the previous framework. This approach usually leads to an isotropic description of damage [10]. Cracking is essentially anisotropic and the model needs to account for it to be realistic in terms of actual damage predictions [11]. The probabilistic approach developed in Section 2 is used to derive the evolution law of the damage variables defined on a continuum level in Section 3.

To represent the real light bilayered armor concept, one of the prevalent test configuration is the entire ceramic/steel specimen. These perforation tests only give partial information, e.g., global displacements or some local stresses. It is also difficult to obtain direct observation of the damage zone evolution during impact and only post-mortem observations can give some indication on the crack density and orientation. An Edge-On Impact (EOI) configuration is a viable alternative to analyze damage state and evolution by recording the crack front pattern and velocity $[12,13]$. A quantitative information on the location and evolution of damage can be obtained. It can be shown that the same damage mechanism (i.e., damage in tension) is observed in EOI experiments and in real impact configurations [11]. In Section 4 different visualization techniques are presented to analyze EOI experiments and to compare them with predictions of the damage model developed in Section 3. 


\section{PROBABILISTIC APPROACH}

The aim of this section is to introduce all microscopic aspects of fracture to describe both static and dynamic loadings. The basis of the approach is an extended version of a Weibull model [14] applicable in both situations. It can be noted that all the material parameters that will be used can be identified by using static tests even in the dynamic range. It will be shown that the key distinction to make is between single and multiple fragmentation rather than static versus dynamic loadings.

\subsection{Single Fragmentation}

The failure of a ceramic in quasi-static tension is due to defects $D_{\mathrm{i}}$ of random location $\underline{x}$ defined by a failure stress $\sigma_{\mathrm{i}}(\underline{x})$. When an equivalent stress $\sigma(\underline{x})$, e.g., maximum principal stress, is greater than $\sigma_{\mathrm{i}}(\underline{x})$, a crack emanating from the defect leads to the failure of the whole structure. Therefore, the ultimate strength of a ceramic specimen is not deterministic and a failure probability $P_{\mathrm{F}}$ must by defined and can be described by a Weibull law [14]

$$
\begin{array}{r}
P_{\mathrm{F}}=1-\exp \left[-\lambda_{\mathrm{t}}(\sigma) Z_{\mathrm{eff}}\right] \\
\lambda_{\mathrm{t}}(\sigma)=\lambda_{0}\left[\frac{\sigma}{S_{0}}\right]^{\mathrm{m}}
\end{array}
$$

where $\lambda_{\mathrm{t}}(\sigma)$ is the defect density, $m$ the Weibull modulus, $S_{0}$ the scale parameter relative to a reference density $\lambda_{0}$ and $Z_{\text {eff }}$ the effective volume, surface or length [15]. It can be noted that the previous formulation enters the framework of a Poisson point process of intensity $\lambda_{\mathrm{t}}(\sigma)$. The mean failure stress $\sigma_{\mathrm{w}}$ 
and the standard deviation $\sigma_{\mathrm{sd}}$ are given by

$$
\sigma_{\mathrm{w}}=\frac{S_{0}}{\left(Z_{\mathrm{eff}} \lambda_{0}\right)^{\frac{1}{\mathrm{~m}}}} \Gamma\left(1+\frac{1}{m}\right), \sigma_{\mathrm{sd}}^{2}=\frac{S_{0}^{2}}{\left(Z_{\mathrm{eff}} \lambda_{0}\right)^{\frac{2}{m}}} \Gamma\left(1+\frac{2}{m}\right)-\sigma_{\mathrm{w}}^{2}
$$

where $\Gamma$ is the Euler function of the second kind. The following study will be concerned with a sintered silicon carbide ceramic ( $\mathrm{SSiC}$ ) made by Céramiques et Composites (France) whose properties are listed in Table 1.

\subsection{Multiple Fragmentation}

In the bulk of an impacted ceramic, damage in tension is observed when the hoop stress induced by the radial motion is sufficiently large to generate fracture in mode I initiating on micro-defects. An example of fragmentation due to high tensile stress rates ( $\mathrm{up}$ to $5.10^{9} \mathrm{MPa} \times \mathrm{s}^{-1}$ ) is presented in Fig. 1 where a ceramic tile is impacted on the edge. The ceramic target presents one mirror polished face illuminated by a flashlight. A high-speed camera records the reflected light [16]. Most of the initiated cracks do not exceed a few millimeters in length (Figs. 1-a/b). This leads us to assume that the crack tip cannot follow the crack front, i.e., the velocity of the former is less than the velocity of the latter. The sharp border line of the damaged zone confirms a very high tensile stress rate: a crack nucleates when the tensile stress reaches a critical value and stops because other defects nucleate in front of it and relax the hoop stresses (Fig. 1-c). The anisotropy due to cracking is also clearly visible.

When a fracture is initiated, the stress state around the propagating crack is a complex function of time, crack velocity and stress wave celerity. In order to simplify the following development, the shape of the affected (or interaction) 
zone (volume, surface or length) $Z_{\mathrm{i}}$ is supposed to be constant, i.e., all the interaction zones are self-similar and $Z_{\mathrm{i}}$ can be written as

$$
Z_{\mathrm{i}}(t-\tau)=S[k C(t-\tau)]^{\mathrm{n}}
$$

where $k C$ is the velocity of a propagating crack, $S$ a shape parameter, $C=$ $(E / \rho)^{1 / 2}$ a stress wave velocity ( $E$ : Young's modulus, $\rho$ : mass density); therefore $k C(t-\tau)$ is a representative length of the relaxation zone at time $t$ around a broken flaw at time $\tau$. The power $n=1,2,3$ is the space dimension. The shape parameter $S$ is defined in order to have $\mathrm{d} \sigma / \mathrm{d} t \leq 0$ in $Z_{\mathrm{i}}$, i.e., no new nucleation can occur in $Z_{\mathrm{i}}$. When the space dimension is $n=3$, it is assumed, as a first approximation, that the obscuration (or shielding) zones are spherical and $S=4 \pi / 3$. By studying the dynamic crack propagation, the value $k=0.38$ is usually assumed [17].

To understand why a crack nucleates, one has to model the interaction of a nucleated defect and other defects that would nucleate. The space location of the defects is represented in a simple abscissa of an $\mathrm{x}-\mathrm{y}$ graph where the y-axis represents time (or stress) to failure of a given defect. In this graph, a shaded cone represents the expansion of the interaction zone with time due to nucleation and propagation of a crack. A section $Z$ (see Fig. 2-a) of a cone can be a volume, a surface or a length, depending on the space dimension $n$. The defects outside the shaded cones can nucleate and produce their own increasing interaction zone (e.g., defects No. 1 and No. 2 of Fig. 2-a). Inside the cones, the defects that should have broken do not nucleate (e.g., defects No. 3 and No. 4 of Fig. 2-a) since they are shielded (or obscured).

Because different interaction volumes may overlap (a flaw can be shielded by one or more cracks), its preferable to define the conditions of non-obscuration 
for a given defect by examining the reverse problem. For a given flaw D, a noninteraction zone can be defined so that, in this zone, a defect cannot obscure D (Fig. 2-b) and the horizon of D in which a defect will always obscure D. The total flaw density $\lambda_{\mathrm{t}}$ can therefore be split into two parts: $\lambda_{\mathrm{b}}$ (the broken flaws) and $\lambda_{\text {obsc }}$ (the obscured flaws). Furthermore, we assume that the distribution of total flaws in a zone $Z$ is modeled by a Poisson point process of intensity $\lambda_{t}[\sigma(t)]$ in accordance with Section 2.1 (Eqn. (2)). New cracks will initiate only if the defect exists in the considered zone and if no defects exist in its horizon

$$
\frac{\mathrm{d} \lambda_{\mathrm{b}}}{\mathrm{d} t}[\sigma(t)]=\frac{\mathrm{d} \lambda_{\mathrm{t}}}{\mathrm{d} t}[\sigma(t)] \times\left[1-P_{\mathrm{o}}(t)\right]
$$

where $P_{\mathrm{o}}$ is the probability of obscuration (i.e., at least one defect is broken in the horizon) expressed as [18-20]

$$
P_{\mathrm{o}}(t)=1-\exp \left\{-\lambda_{\mathrm{t}}[\sigma(t)] \mathcal{Z}(t)\right\}
$$

where $\mathcal{Z}(t)$ denotes the mean obscuration zone which is calculated by averaging at time $t$ the section of the obscured zones $Z_{\mathrm{i}}(t-\tau)$ for a nucleation at time $\tau$ and with a density $\frac{1}{\lambda_{\mathrm{t}}[\sigma(t)]} \frac{\mathrm{d} \lambda_{\mathrm{t}}}{\mathrm{d} t}[\sigma(\tau)]$

$$
\mathcal{Z}(t) \lambda_{\mathrm{t}}[\sigma(t)]=\int_{0}^{t} \frac{\mathrm{d} \lambda_{\mathrm{t}}}{\mathrm{d} t}[\sigma(\tau)] Z_{\mathrm{i}}(t-\tau) \mathrm{d} \tau
$$

The similarity of Eqns. (1) and (6) shows a first link between single and multiple fragmentation if one remembers that, for very low stress rates, the mean obscured zone $\mathcal{Z}(t)$ has to be limited by the volume, surface or length $Z$ of the body. The Weibull law is recovered from the fragmentation model when the entire structure is obscured by only one broken defect. 


\subsection{Characteristic Parameters and Scalings}

When dynamic loadings are considered with a constant stress rate $\mathrm{d} \sigma / \mathrm{d} t=\dot{\sigma}$, one can define a dimensionless flaw density $\left(\bar{\lambda}=\lambda / \lambda_{\mathrm{c}}\right)$, time $\left(\bar{t}=t / t_{\mathrm{c}}\right)$, space measure $\left(\bar{Z}=Z / Z_{\mathrm{c}}\right)$ and stress $\left(\bar{\sigma}=\sigma / \sigma_{\mathrm{c}}\right)$ from the condition

$$
\lambda_{\mathrm{c}} Z_{\mathrm{c}}=1 \text { with } \lambda_{\mathrm{c}}=\lambda_{\mathrm{t}}\left[\sigma\left(t_{\mathrm{c}}\right)\right] \text { and } Z_{\mathrm{c}}=Z_{\mathrm{i}}\left(t_{\mathrm{c}}\right)
$$

where the subscript ' $c$ ' denotes characteristic quantities. A characteristic stress is defined by $\sigma_{\mathrm{c}}=\dot{\sigma} t_{\mathrm{c}}$. Equation (8) expresses the fact that the characteristic zone of measure $Z_{\mathrm{c}}$ contains on average a flaw that may break at the characteristic time $t_{\mathrm{c}}$. The characteristic parameters are given by

$$
\begin{gathered}
t_{\mathrm{c}}=\left[\frac{S_{0}^{\mathrm{m}}}{\lambda_{0} S(k C)^{\mathrm{n}} \dot{\sigma}^{\mathrm{m}}}\right]^{\frac{1}{\mathrm{~m}+\mathrm{n}}}, Z_{\mathrm{c}}=\left[\frac{\left(S_{0} k C\right)^{\mathrm{m}} S^{\mathrm{m} / \mathrm{n}}}{\lambda_{0} \dot{\sigma}^{\mathrm{m}}}\right]^{\frac{\mathrm{n}}{\mathrm{m}+\mathrm{n}}}, \\
\sigma_{\mathrm{c}}=\left[\frac{S_{0}^{\mathrm{m}} \dot{\sigma}^{\mathrm{n}}}{\lambda_{0} S(k C)^{\mathrm{n}}}\right]^{\frac{1}{\mathrm{~m}+\mathrm{n}}} .
\end{gathered}
$$

By using Eqns. (2), (4) and (6) a closed-form solution is proposed for the differential equation (5) in the case of a constant stress rate $\dot{\sigma}$

$$
\bar{\lambda}_{\mathrm{b}}(\bar{\sigma})=\frac{m}{m+n}\left(\begin{array}{c}
m+n \\
m
\end{array}\right)^{\frac{m}{m+n}} \gamma\left[\frac{m}{m+n}, \frac{\bar{\sigma}^{\mathrm{m}+\mathrm{n}}}{\left(\begin{array}{c}
m+n \\
m
\end{array}\right)}\right]
$$

where $\gamma[p, x]=\int_{0}^{x} t^{\mathrm{p}-1} \exp (-t) \mathrm{d} t$ is the incomplete gamma function and $\left(\begin{array}{c}m+n \\ m\end{array}\right)$ a binomial coefficient.

The variable $P_{\mathrm{o}}$ can be used to define a damage variable in the framework of Continuum Damage Mechanics. By averaging over a representative zone (to be specified later on), $P_{\mathrm{o}}$ is equal to the damage variable $D$, with $D=0$ for the virgin material and $D=1$ for the fully broken one. It is interesting to 
notice that the first order approximation of Eqn. (6) leads to the differential equation proposed by Grady and Kipp [21] to describe the evolution of a damage variable. By using Eqns. (2), (4) and (6), the evolution of the damage parameter is written as

$$
D=1-\exp \left[-\frac{(\bar{\sigma})^{\mathrm{m}+\mathrm{n}}}{\left(\begin{array}{c}
m+n \\
m
\end{array}\right)}\right] .
$$

Equation (11) shows that $D(\bar{\sigma}=1) \cong 0$ and $D(\bar{\sigma}=2) \cong 1$ (i.e., most of the damage evolution occurs during a time interval equal to $t_{\mathrm{c}}$ ). During $t_{\mathrm{c}}$, the measure of the horizon is limited by $Z_{\mathrm{i}}\left(t_{\mathrm{c}}\right)=Z_{\mathrm{c}}$ therefore the minimum measure of the representative zone is $Z_{\mathrm{c}}$. By noting that the applied stress $\Sigma$ is related to the local (or effective) stress $\sigma$ by $\sigma=\Sigma /(1-D)[22]$, the ultimate strength $(d \Sigma / d \sigma=0)$, denoted by $\Sigma_{\max }$, is expressed as

$$
\frac{\Sigma_{\max }}{\sigma_{\mathrm{c}}}=\left[\frac{1}{\mathrm{e}} \frac{\left(\begin{array}{l}
m \\
m
\end{array}\right)}{(m+n)}\right]^{\frac{1}{\mathrm{~m}+\mathrm{n}}} .
$$

\subsection{Single / Multiple Fragmentation}

The present section is devoted to the analysis of the transition between single and multiple fragmentation. Figure 3 shows the evolution of the ultimate stress vs. stress rate in a volume $Z$ equal to $Z_{\text {eff }}=1.25 \mathrm{~mm}^{3}$ (see Table 1 ). The lines represent analytical solutions while the dots and error bars represent Monte-Carlo simulations of an underlying Poisson point process. For a stress rate within $\left[0,5 \times 10^{8} \mathrm{MPa} \times \mathrm{s}^{-1}\right]$, the ultimate stress is not modified by

the loading rate. When $\dot{\sigma}$ increases of approximately one order of magnitude, the ultimate strength follows the proposed solution (Eqn. (12)). During the single / multiple fragmentation transition, the difference between the solid lines (Eqns. (3) and (12)) and the Monte-Carlo simulations does not exceed 
10\%. The standard deviation significantly decreases in the case of multiple fragmentation when the stress rate increases. Even if the ultimate strength has to be defined for both static and dynamic loadings by a mean value and a standard deviation, one can see that dynamic loadings lead to a more 'deterministic' behavior in the multiple fragmentation regime. Furthermore, for $\mathrm{SSiC}$ ceramics, a stress rate up to $10^{7} \mathrm{MPa} \times \mathrm{s}^{-1}$ has shown no stress rate effect on the mean strength [23]. This observation is in good agreement with the result shown in Fig. 3.

The transition between single and multiple fragmentation can be estimated by the intersection between the weakest link and the multiple fragmentation solutions (see Fig. 3)

$$
\sigma_{\mathrm{w}}=\Sigma_{\max }(\dot{\sigma})
$$

The transition defined by Eqn. (13) leads to the following inequalities

$$
\begin{cases}\frac{Z}{Z_{\mathrm{c}}(\dot{\sigma})}<g(m) & \text { Single fragmentation } \\ \frac{Z}{Z_{\mathrm{c}}(\dot{\sigma})} \geq g(m) \quad \text { Multiple fragmentation }\end{cases}
$$

with

$$
g(m)=\left[\mathrm{e} \frac{(m+n)}{\left(\begin{array}{c}
m+n \\
m
\end{array}\right)}\right]^{\frac{\mathrm{m}}{\mathrm{m}+\mathrm{n}}} \Gamma\left(1+\frac{1}{m}\right)^{\mathrm{m}}
$$

The size $Z_{\mathrm{c}}$ can therefore be considered as the characteristic scale for which a single / multiple fragmentation transition is observable. This conclusion is consistent with the analysis following the damage kinetic law (Eqn. (11)). Furthermore, Fig. 3 shows that when $Z / Z_{\mathrm{c}} \geq 1$ the ultimate strength scatter is very small. This characteristic volume can be used in FE computations in 
which the mesh size has to be greater than or equal to $Z_{\mathrm{c}}$ to use a continuum (and deterministic) description of damage. It is worth mentioning that this transition does not only depend upon material (Weibull) parameters but also involves the size $Z$ of the considered structure and the applied stress rate $\dot{\sigma}$. The response of a large structure can be considered as 'dynamic' for low stress rate even if the same material follows the weakest link hypothesis for the same loading applied on a smaller volume. There is therefore no intrinsic relationship between material parameters and characteristic scales to describe the fragmentation of brittle materials.

The size $Z_{\mathrm{c}}$ can also be used to check the mesh-relevance of a finite element calculation. During a time step, the variables (such as nucleated flaws density, damage) are computed by using their past evolution over the horizon, as mentioned in Section 2.2. An hypothesis of uniformity of the variables over this horizon is therefore made. When the mesh size is smaller than the horizon, two contiguous Gauss points (i.e., two neighboring elements in the present case) have their horizons overlapping: a space location may have two different sets of variables. To avoid such a situation, the minimum mesh size must be greater than or equal to the horizon, which is limited by the characteristic zone $Z_{\mathrm{c}}$. The minimum mesh size is dependent on material properties and on the loading condition: the higher the stress rate, the smaller the mesh size. This is consistent with the general practice of mesh refining when shock waves are suspected to occur.

Lastly, the size $Z_{\mathrm{c}}$ may also be used to know whether a continuum or a discrete approach applies. For low stress rates in the structure, few fragments are generated (i.e., $Z<g(m) Z_{\mathrm{c}}$ ) and a discrete model has therefore to be used (e.g., [2]). Conversely, when high stress rates are generated, (i.e., $Z>g(m) Z_{\mathrm{c}}$ : 
many small fragments are produced) the discrete method leads to calculations of fragmented structures beyond the limits of nowadays computers. A continuum approach can be used to recover the microstructure (fracture density and orientation) generated during the impact.

\section{DAMAGE MODEL}

In Section 2.4, it was shown that a continuum description of damage can be used when the impact stress rate is large enough to generate multiple fragmentation. Under this assumption, an anisotropic damage model is now developed.

\subsection{Damage Description and Kinetic Law}

The analysis of Section 2.2 is extended to account for three-dimensional situations. The proposed damage variable is defined with the assumption that many cracks nucleate and propagate due to a tensile stress expressed in the direction of the maximum principal stress. Since the cracks will be strongly oriented, an anisotropic damage description is chosen [11]. It is assumed that the damage directions coincide with the principal stress directions (i.e., mode I mechanism). The anisotropic description is expressed through a second order damage tensor by assuming that the cracks remain open so that the energy contribution of higher order terms can be neglected $[24,25]$. The damage tensor $\underline{\underline{D}}$ is diagonal in the eigen directions of $\underline{\underline{\sigma}}$ and only 3 variables $\left(D_{1}, D_{2}\right.$, $\left.D_{3}\right)$ have to be computed. The relationship between the microscopic principal 
stress $\sigma_{\mathrm{i}}$ and the macroscopic one $\left(\Sigma_{\mathrm{i}}\right)$ is $[22,26]$

$$
\sigma_{\mathrm{i}}=\frac{\Sigma_{\mathrm{i}}}{1-D_{\mathrm{i}}} \text { with } \mathrm{i}=1,2,3
$$

The state potential is assumed to be given by the Gibbs' specific enthalpy $\rho \Phi$. It is expressed as a function of the macroscopic stress tensor $\cong$ and the damage variables $D_{1}, D_{2}$ and $D_{3}$

$$
\rho \Phi=\frac{1}{2} \underline{\underline{\underline{\Sigma}}}: \underline{\underline{\underline{S}}}\left(D_{1}, D_{2}, D_{3}\right): \underline{\underline{\Sigma}}
$$

where $\underline{\underline{\underline{\underline{S}}}}$ is the compliance tensor dependent upon $D_{1}, D_{2}$ and $D_{3}$ and ':' the contraction wrt. two indices. The associated forces are defined as

$$
\begin{aligned}
& \underline{\underline{E}}=\rho \frac{\partial \Phi}{\partial \underline{\underline{\underline{\Sigma}}}}=\underline{\underline{\underline{\underline{S}}}}\left(D_{1}, D_{2}, D_{3}\right): \underline{\underline{\underline{\Sigma}}} \\
& Y_{\mathrm{i}}=\rho \frac{\partial \Phi}{\partial D_{\mathrm{i}}}=\frac{1}{2} \underline{\underline{\underline{\Sigma}}}: \frac{\partial \underline{\underline{\underline{\underline{\underline{S}}}}}}{\partial D_{\mathrm{i}}}\left(D_{1}, D_{2}, D_{3}\right): \underline{\underline{\underline{\Sigma}}}
\end{aligned}
$$

where $\underline{\underline{E}}$ denotes the strain tensor and $Y_{\mathrm{i}}$ the energy release rate density associated to the damage variable $D_{\mathrm{i}}$. By using Linear Elastic Fracture Mechanics, one can show that cracking in mode I induces only a compliance increase in the normal direction to the crack plane [27]. Therefore, in the principal stress frame (assumed to coincide with the principal damage frame), Eqn. (18) can be written as

$$
\left[\begin{array}{l}
E_{1} \\
E_{2} \\
E_{3}
\end{array}\right]=\frac{1}{E}\left[\begin{array}{ccc}
\frac{1}{1-D_{1}} & -\nu & -\nu \\
-\nu & \frac{1}{1-D_{2}} & -\nu \\
-\nu & -\nu & \frac{1}{1-D_{3}}
\end{array}\right]\left[\begin{array}{c}
\Sigma_{1} \\
\Sigma_{2} \\
\Sigma_{3}
\end{array}\right]
$$


where $E_{1}, E_{2}, E_{3}$ are the principal strains and $\nu$ the Poisson's ratio of the virgin material. Similarly, Eqn. (19) yields

$$
Y_{\mathrm{i}}=\frac{\sigma_{\mathrm{i}}^{2}}{2 E}
$$

The evolution of $D_{\mathrm{i}}$ is expressed in a differential form in order to be implemented in the FE code PamShock [28] by using Eqns. (2), (4), (6) and (21)

$$
\frac{\mathrm{d}^{\mathrm{n}-1}}{\mathrm{~d} t^{\mathrm{n}-1}}\left(\frac{1}{1-D_{\mathrm{i}}} \frac{\mathrm{d} D_{\mathrm{i}}}{\mathrm{d} t}\right)=\lambda_{\mathrm{t}}\left(\sqrt{2 E Y_{\mathrm{i}}}\right) n ! S(k C)^{\mathrm{n}} \text { when } \frac{\mathrm{d} Y_{\mathrm{i}}}{\mathrm{d} t}>0
$$

According to classical results of Continuum Damage Mechanics, the evolution of $D_{\mathrm{i}}$ is stopped if $\mathrm{d} Y_{\mathrm{i}} / \mathrm{d} t<0$. The directions $\left(\underline{d}_{1}, \underline{d}_{2}, \underline{d}_{3}\right)$ associated to $D_{1}$, $D_{2}$ and $D_{3}$ may change at each time step until $D_{1}$ reaches a threshold value $D_{\text {th }}=0.01$. Only the direction $\underline{d}_{1}$ is then locked, the other directions follow the eigen directions of $\underline{\underline{\sigma}}$, with the constraint to be perpendicular to $\underline{d}_{1}$. When $D_{2}$ reaches the threshold value, the whole directions $d_{\mathrm{i}}$ are locked.

\subsection{Mesh Size Dependence}

To test the mesh-sensitivity of the above-described model, one may consider a plane shock wave spall configuration. When a compressive stress pulse is generated in a plate, the reflected (tensile) pulse traveling back from the free rear surface of the specimen is superimposed on the loading pulse. When the loading pulse duration is properly prescribed, a tensile stress is generated in the bulk of the specimen. The thickness of the damaged zone may strongly vary if the model is mesh-dependent. A one-dimensional code [11] is used with numerical viscosity parameters chosen in order to preserve the loading pulse shape during propagation. The plate thickness is $10 \mathrm{~mm}$ and the imposed 
pressure is $-1 \mathrm{GPa}$, for a duration of $10 \mu \mathrm{s}$. Four different mesh densities are tested: $20 \mathrm{el} . / \mathrm{mm}, 40 \mathrm{el} . / \mathrm{mm}, 80 \mathrm{el} . / \mathrm{mm}, 160 \mathrm{el} . / \mathrm{mm}$. The results presented in Fig. 4 show no dependence of the size of the damaged zone on the mesh density.

\section{EXPERIMENTS AND COMPUTATIONS}

Tensile cracking, one of the major degradation mechanisms during interaction, can be observed during impact by using the Edge On Impact configurations instead of a real configuration where the degradation is 'hidden' in the bulk of the ceramic. These configurations are developed by the Ernst-Mach-Institut (EMI) in Germany $[12,29]$ and by the Centre Technique d'Arcueil (CTA) in France $[13,16]$. In the latter configuration, a blunt steel projectile (11 $\mathrm{mm}$ in diameter and $20 \mathrm{~mm}$ in length) impacts a ceramic tile of size $100 \times 50 \times 10 \mathrm{~mm}^{3}$.

A first analysis is an EOI with an aluminum confinement (called sarcophagus) presented in Fig. 5-a. After impact, the tile is coated in an epoxy resin and polished for macroscopic and microscopic analyses. The post-mortem crack pattern is presented in Figs. 5-b/c. Different zones can be separated with respect to the crack density and orientation. In front of the projectile, a small zone exhibits a randomly oriented crack pattern. In the bulk of the ceramic, one can observe long radial cracks with a second circumferential crack pattern superimposed on the first one. In this last region, some radial cracks seem to kink in the circumferential direction, kink one more time and propagate in the radial direction. The kinked fractures are made of two small (and hardly visible, see Fig. 5-c) cracks linked by a small circumferential crack. This complex crack pattern is then opened by the radial motion of the ceramic, widens 
and becomes a long macroscopic 'kinked' fracture. This phenomenon shows that the radial cracks appear prior to the circumferential ones, since the latter does not go through the former. Near the tile rear face, a third zone exhibits a high density of cracks. This thin strip remains at a constant distance from the rear surface, like a spalling zone. It is worth mentioning that the cracks remain in the radial and circumferential directions even if this zone is parallel to the rear surface. The numerical prediction is presented in Fig. 5-d. The cracking mechanism can be described as the superposition of a radially- and a circumferientially-oriented crack pattern, the former with a higher density than the latter. The radially-oriented crack pattern is generated during the first micro-seconds of impact. It is followed by the circumferential ones due to the relaxation wave emanating from the projectile and the rear face of the ceramic tile. This result is in good accordance with the experimental observations. The zone in front of the projectile in which only few radial cracks seem to have nucleated is well reproduced. Finally, the high density crack zone looking like a spalling region is also well described, with a crack direction approximately in the radial and circumferential direction, as observed in the sarcophagus configuration.

A second utilization of the EOI configuration provides quantitative strain measurements over a field of $32 \times 32 \mathrm{~mm}^{2}$ during impact. A Moiré photography set-up has been developed [30]. A typical result of the high-speed Moiré photography is presented in Fig. 6 with an impactor velocity equal to $330 \mathrm{~m} / \mathrm{s}$. The method used to analyze the fringes cannot give reliable data when they are blurred. To overcome this problem, the artifacts generated during the fringe pattern analysis are automatically reset to zero in a gray colored zone in Figs. 6-c/d. Figure 6-a is the fringe pattern approximately $2 \mu$ s after impact. Figure 6-b shows the eigen directions of the third (compressive) strain 
and confirms that the strain wave induced by an EOI has a circular geometry. The maximum and minimum eigen strains are plotted in Figs. 6-c and $6-\mathrm{d}$, respectively. The advantage of the Moiré measurement is that a quantitative rather than qualitative analysis can be performed between experiments and simulations. To compare the evolution of the radial and hoop strains, a typical result is given in Fig. 7. The strain diagram is plotted for a point $M$ at a distance of $13 \mathrm{~mm}$ from the surface hit by the projectile (see Fig. 6-a). It can be noticed that the radial strain reaches an important value before any significant evolution of the hoop strain. This is consistent with a cylindrical stress wave in which the tensile strain is induced by the radial motion of the material. The general shape of the curve is very well reproduced by the model, with a tendency to under-estimate the compressive strain. A better modeling of the compressive behavior of the ceramic should improve the numerical/experimental agreement.

\section{CONCLUSION}

A probabilistic approach has been proposed to describe the stress rate dependence of the fragmentation mechanism. Based upon the probabilistic approach, a damage evolution law in tensile mode is derived for impacted ceramics within the framework of Continuum Mechanics. The material parameters used in the model are identified through quasi-static 3-point flexural tests. A closed-form solution for the number of cracks nucleated is proposed and leads to the definition of the transition between single and multiple fragmentation. The probabilistic nature of quasi-static failure progressively vanishes with high stress rates and therefore allows a continuum description of fragmentation by using damage variables. Characteristic quantities (such as volume or time) are 
proposed to choose the mesh size used in numerical simulations. A spalling experiment is analyzed to investigate the influence of mesh size on the shape of the damaged zone. No mesh sensitivity is observed. The model can provide an estimation of the number and directions of activated flaws as well as anisotropic damage variables. Complex damage patterns, in which superposition of cracks of different directions occurs, can be computed. The strains of an impacted ceramic can also be predicted with a good accuracy, even if the ceramic becomes fully fragmented.

\section{ACKNOWLEDGMENTS}

The authors wish to thank Mr. A. Trameçon from ESI for his valuable help in implementing the model in PamShock.

\section{References}

[1] P. C. den Reijer, Impact on Ceramic Faced Armor, (PhD dissertation, Delft Technical University, 1991).

[2] G. T. Camacho and M. Ortiz, Computational Modelling of Impact Damage in Brittle Materials, Int. J. Solids Struct. 33 [20-22] (1996) 2899-2938.

[3] J. J. Monaghan, Smoothed Particle Hydrodynamics, Annu. Rev. Astron. Astrophys. 30 (1992) 543-574.

[4] L. D. Libersky and A. G. Petscheck, High Strain Lagrangian Hydrodynamics, J. Comp. Phys. 109 (1993) 67-75. 
[5] S. Hiermaier and W. Riedel, Numerical Simulation of Failure in Brittle Materials using Smooth Particle Hydrodynamics, Proceedings New Models and Numerical codes for Shock Wave Processes in Condensed Media, 1997.

[6] B. Budiansky and R. J. O'Connell, Elastic Moduli of a Cracked System, Int. J. Solids Struct. 12 (1976) 81-97.

[7] L. G. Margolin, Elasticity Moduli of a Cracked Body, Int. J. Fract. 22 (1983) 65-79.

[8] L. M. Taylor, E.-P. Chen and J. S. Kuszmaul, Microcrack-Induced Damage Accumulation in brittle Rocks under Dynamic Loading, Comp. Meth. Appl. Mech. Eng. 55 (1986) 301-320.

[9] A. M. Rajendran, Modeling the Impact Behavior of AD85 Ceramic under Multiaxial Loading, Int. J. Impact Eng. 15 [6] (1994) 749-768.

[10] J. Lemaitre, A Course on Damage Mechanics, (Springer-Verlag, Berlin (Germany), 1992).

[11] C. Denoual, C. E. Cottenot and F. Hild, On the Identification of Damage during Impact of a Ceramic by a Hard Projectile, Proceedings 16th International Conference on BALLISTICS, (APDS, Arlington, VA (USA), 1996), 541-550.

[12] E. Strassburger, H. Senf and H. Rothenhausler, Fracture Propagation during Impact in Three Types of Ceramics, J. Physique IV coll. C8 [suppl. IV] (1994) 653-658.

[13] P. Riou, Contribution l'étude de l'endommagement du carbure de silicium lors d'un impact de basse énergie : application aux blindages, (PhD dissertation, Ecole Nationale Supérieure des Mines de Paris, 1996).

[14] W. Weibull, A Statistical Theory of the Strength of Materials, (Roy. Swed. Inst. Eng. Res., 151, 1939). 
[15] D. G. S. Davies, The Statistical Approach to Engineering Design in Ceramics, Proc. Brit. Ceram. Soc. 22 (1973) 429-452.

[16] P. Riou, C. Denoual and C. E. Cottenot, Visualization of the Damage Evolution in Impacted Ceramic Carbide Ceramics, Int. J. Impact Eng. 21 [4] (1998) 225235 .

[17] M. F. Kanninen and C. H. Popelar, Advanced Fracture Mechanics, (Oxford University Press, New-York, NY (USA), 1985).

[18] J. Serra, Image Analysis and Mathematical Morphology, (Academic Press, London (UK), 1982).

[19] D. Jeulin, Anisotropic Rough Surface Modeling by Random Morphological Functions, Acta Stereol. 6 (1987) 183-189.

[20] C. Denoual, G. Barbier and F. Hild, A Probabilistic Approach for Fragmentation of Ceramics under Impact Loading, C. R. Acad. Sci. Paris 325 [Srie IIb] (1997) 685-691.

[21] D. E. Grady and M. E. Kipp, Continuum Modeling of Explosive Fracture in Oil Shale, Int. J. Rock Min. Sci. Geomech. 17 (1980) 147-157.

[22] Y. N. Rabotnov, Creep Problems in Structural Members, (North-Holland, Amsterdam (the Netherlands), 1969).

[23] C. Denoual and P. Riou, Comportement à l'impact de céramiques techniques pour blindages légers, (CREA, Report 95 R 005, 1995).

[24] M. Kachanov, Elastic Solids with Many Cracks and Related Problems, Adv. Appl. Mech. 30 (1994) 259-445.

[25] A. Dragon and D. Halm, A Model of Anisotropic Damage by Macrocrack Growth; Unilateral Effect, in: D. Jeulin, ed., Proceedings Workshop on the 'Mechanical Behavior of Damaged Solids', (Fontainebleau (France), 1995). 
[26] S. Murakami and N. Ohno, A Continuum Theory of Creep and Creep Damage, in: A. R. S. Ponter and D. R. Hayhurst, eds., Proceedings Creep in Structures, (Springer, Berlin (Germany), 1981), 422-444.

[27] A. Burr, F. Hild and F. A. Leckie, Micro-Mechanics and Continuum Damage Mechanics, Arch. Appl. Mech. 65 [7] (1995) 437-456.

[28] Pamshock, User's Manual, ESI (1996)

[29] E. Strassburger and H. Senf, Experimental Investigations of Wave and Fracture Phenomena in Impacted Ceramics and Glasses, (ARL, Report ARL-CR-214, 1995).

[30] T. Bertin-Mourot, C. Denoual, G. Dehors, P.-F. Louvign and T. Thomas, High Speed Photography of Moiré Fringes - Application to Ceramics under Impact, J. Physique IV Coll. C3 [Suppl. III] (1997) 311-316. 
Table 1

Material properties of a SSiC ceramic.

\begin{tabular}{|c|c|}
\hline Property & $\mathrm{SSiC}$ \\
\hline Young's Modulus (GPa) & 410 \\
\hline Density & 3.15 \\
\hline Weibull Modulus & 9.6 \\
\hline Mean Strength (MPa) & 360 \\
\hline Effective Volume $\left(\mathrm{mm}^{3}\right)$ & 1.25 \\
\hline
\end{tabular}




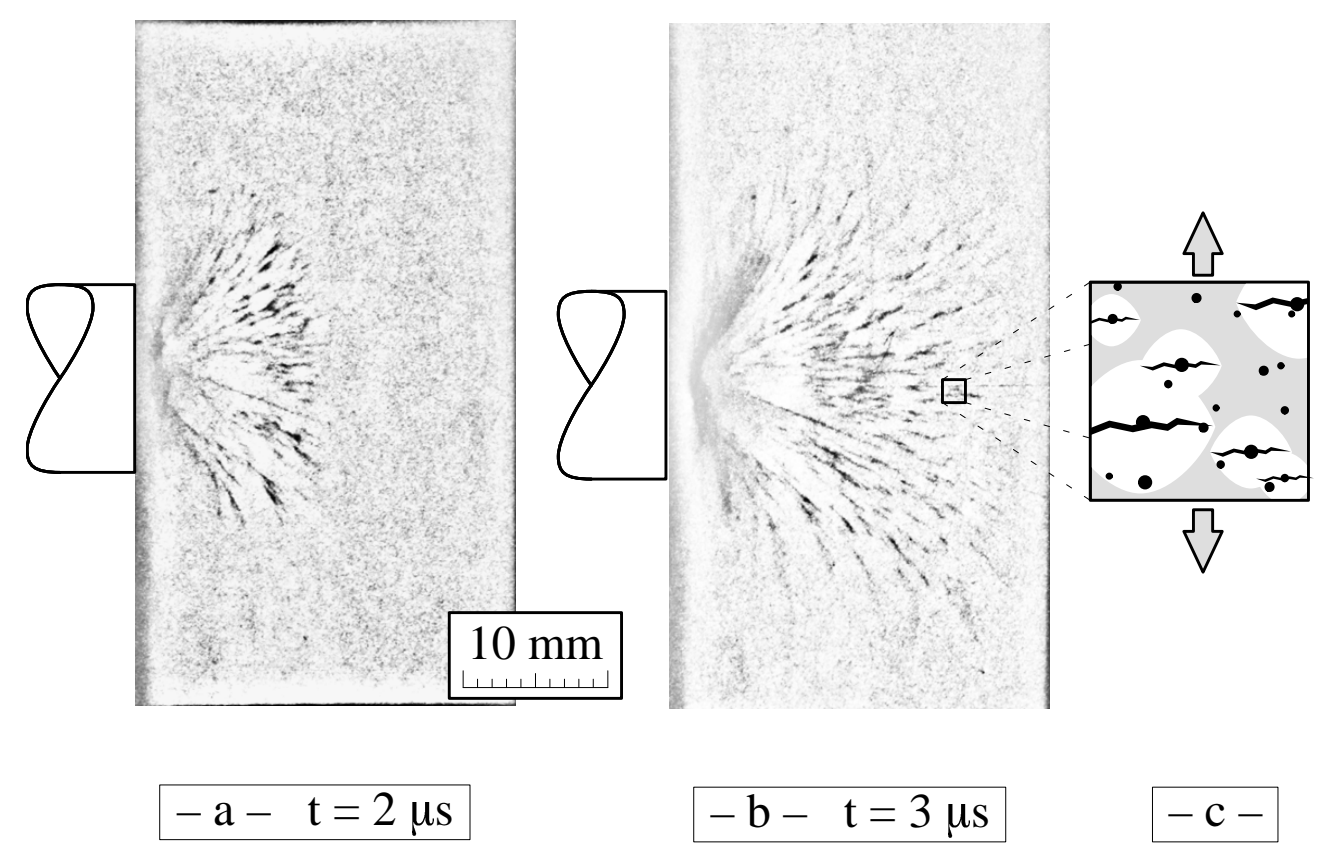

Fig. 1. a-, b-An example of pictures obtained by real-time visualization of a SSiC ceramic impacted at a velocity of $203 \mathrm{~m} / \mathrm{s}$ (after [13]).

c-Depiction of the obscuration mechanism (the white zones represent relaxed areas where no new cracks can nucleate). 

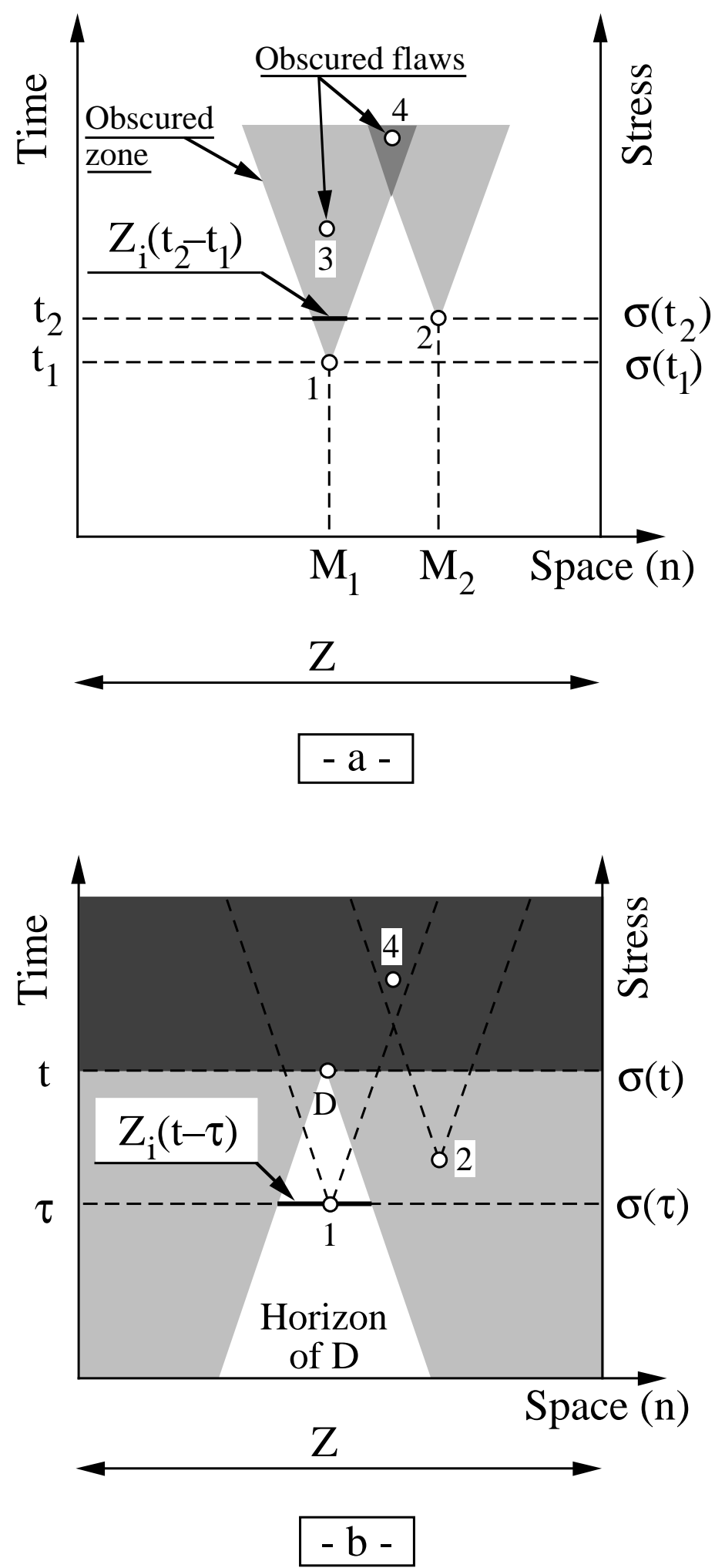

Fig. 2. a-Depiction of obscuration phenomena.

b-Schematic of the obscuration/non-obscuration zones for a defect D. 
Single Fragmentation : Multiple Fragmentation

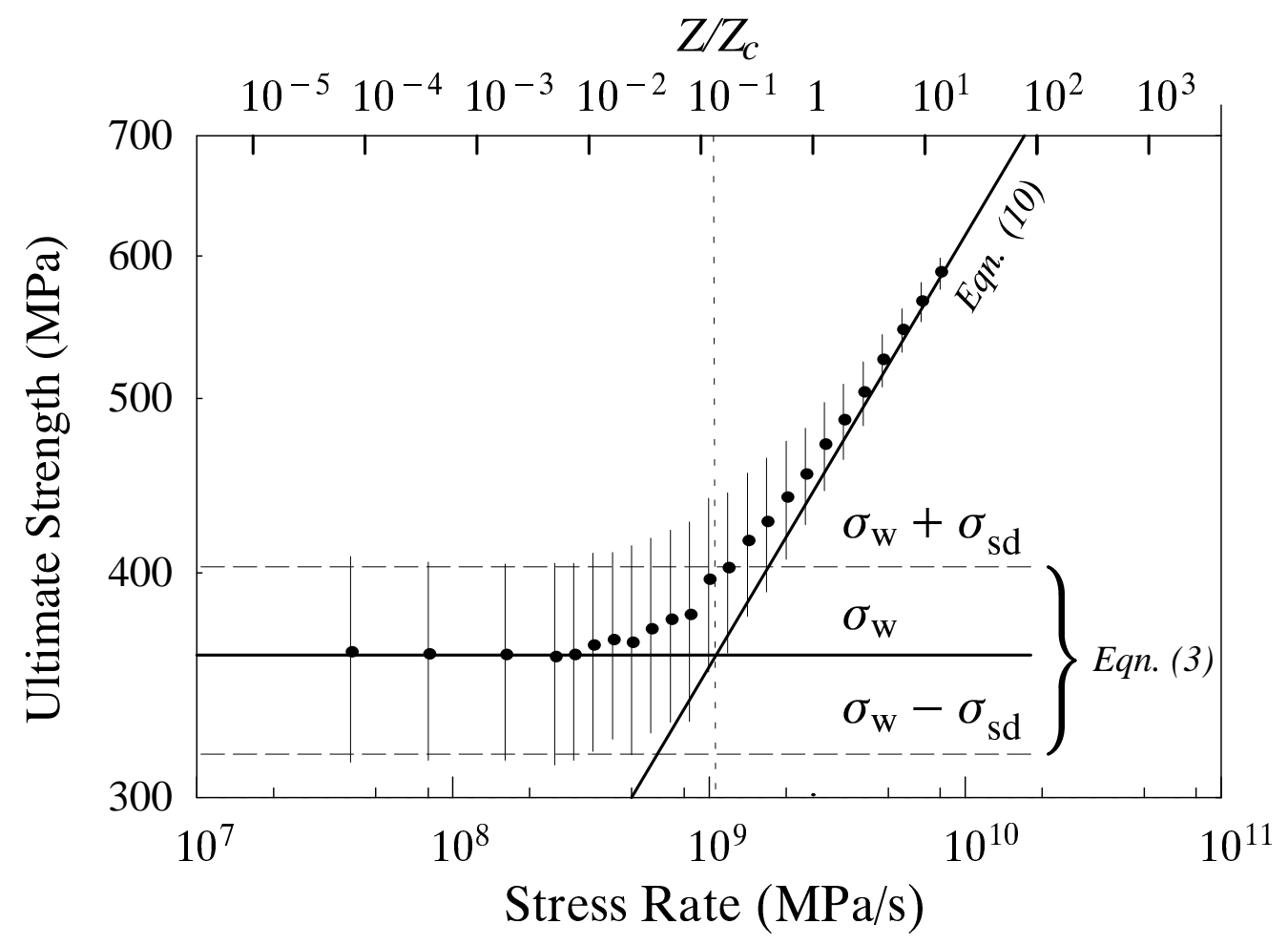

Fig. 3. Ultimate strength vs. stress rate (SSiC). The dots and error bars represent results obtained by Monte-Carlo simulations (500 realizations/point) and their standard deviation when $Z=Z_{\text {eff }}$ and $n=3$. 


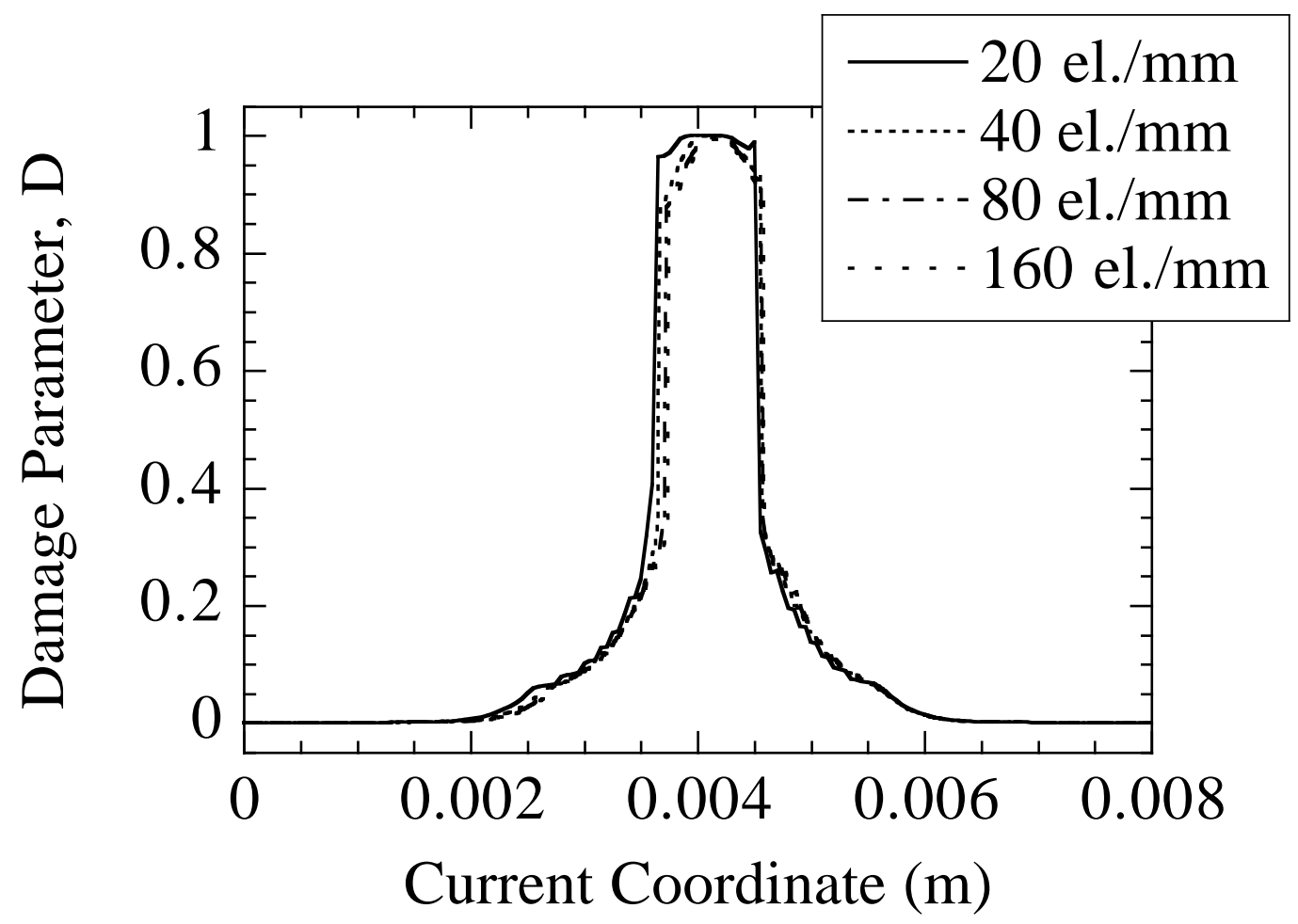

Fig. 4. Damage location for 4 mesh densities in a plane shock wave spall configuration $(\mathrm{SSiC})$. 


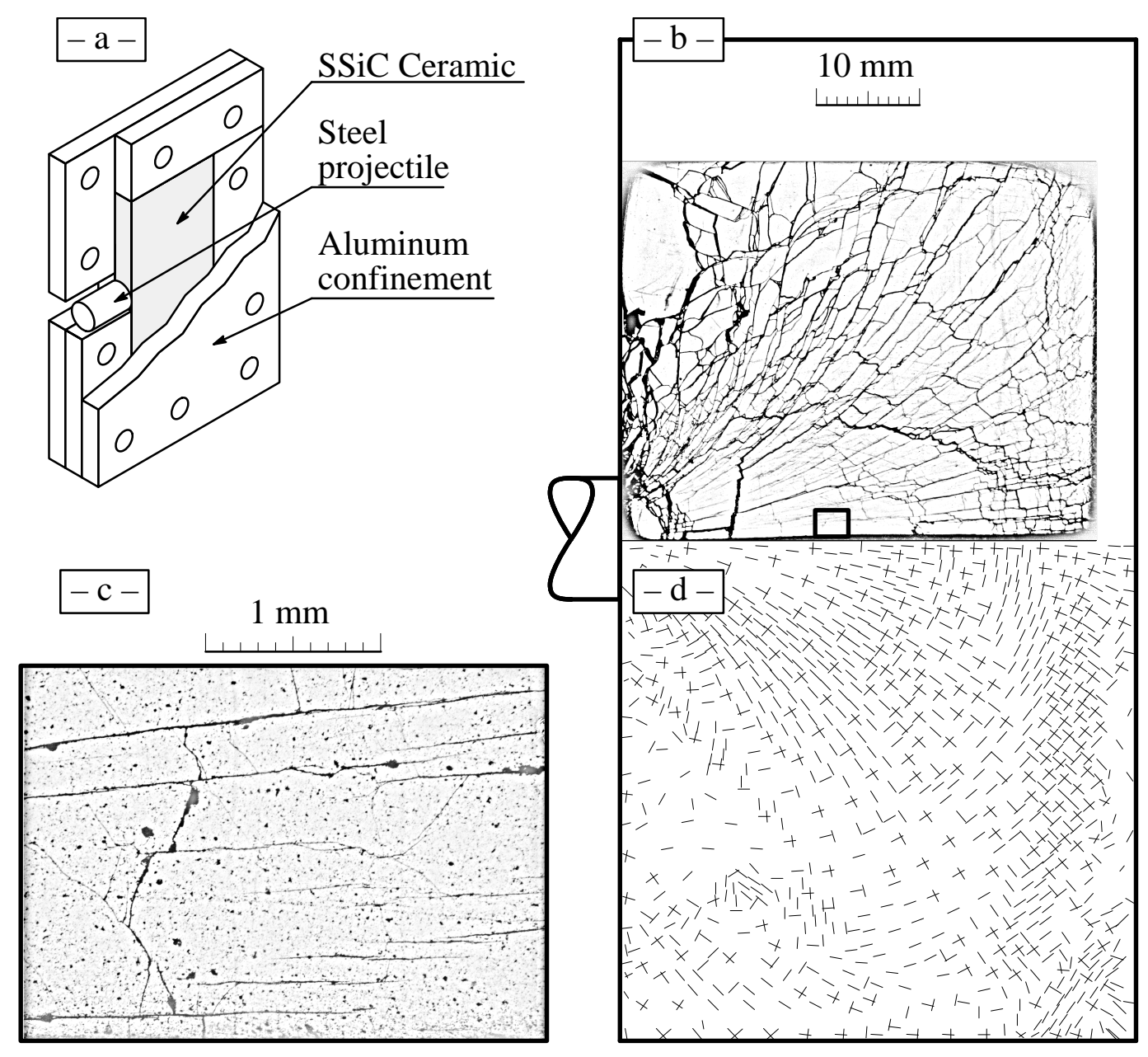

Fig. 5. a-Schematic of the ceramic confinement. The tile is confined between two $10 \mathrm{~mm}$ thick aluminum plates. The edge confinement is obtained by aluminum tiles screwed on the plates.

b-Half top view of tile made of $\mathrm{SSiC}$ impacted at a velocity of $330 \mathrm{~m} / \mathrm{s}$.

c-The magnification (of the boxed area of -b-) shows many short radial cracks linked by short circumferential ones.

d-Half bottom view of the corresponding numerical simulation. The lines depict the crack directions (perpendicular to the direction of the associated damage). The number of cracks is dependent on the nucleated defect density. 

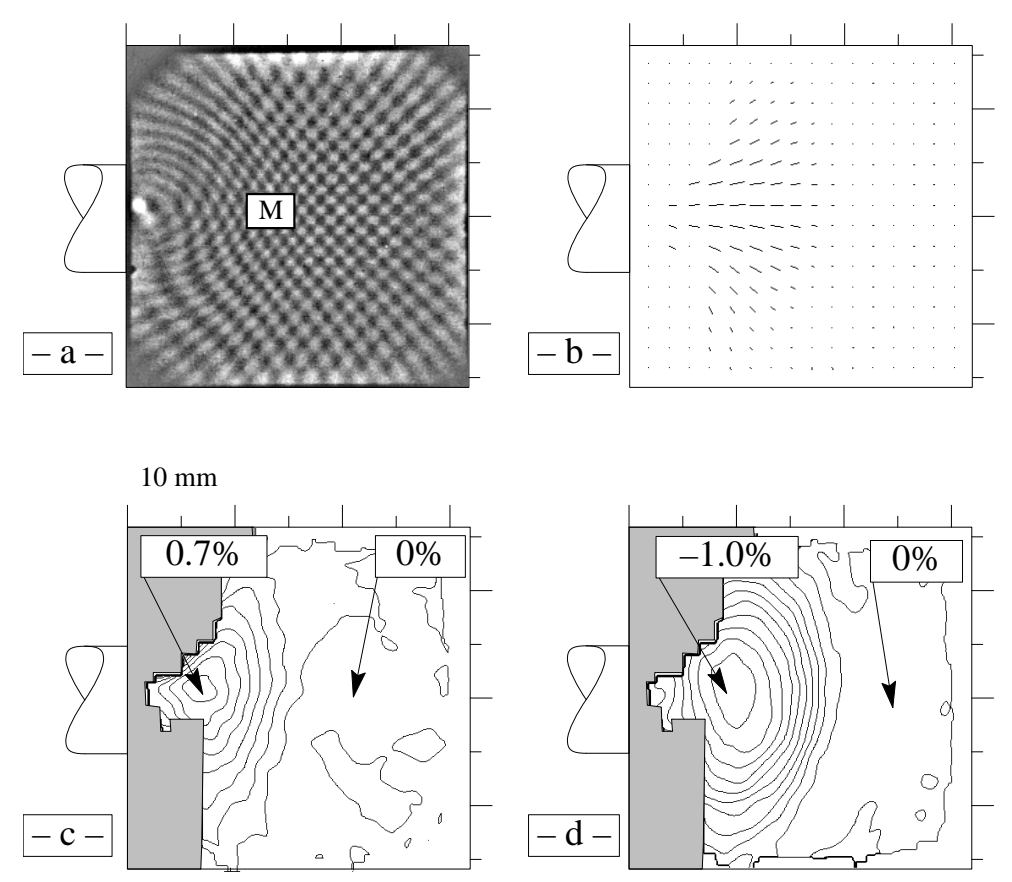

Fig. 6. a-The initial Moiré frame (2 $\mu$ s after impact).

b-Third (negative) eigen direction.

c-and d-First and third eigen strains. 


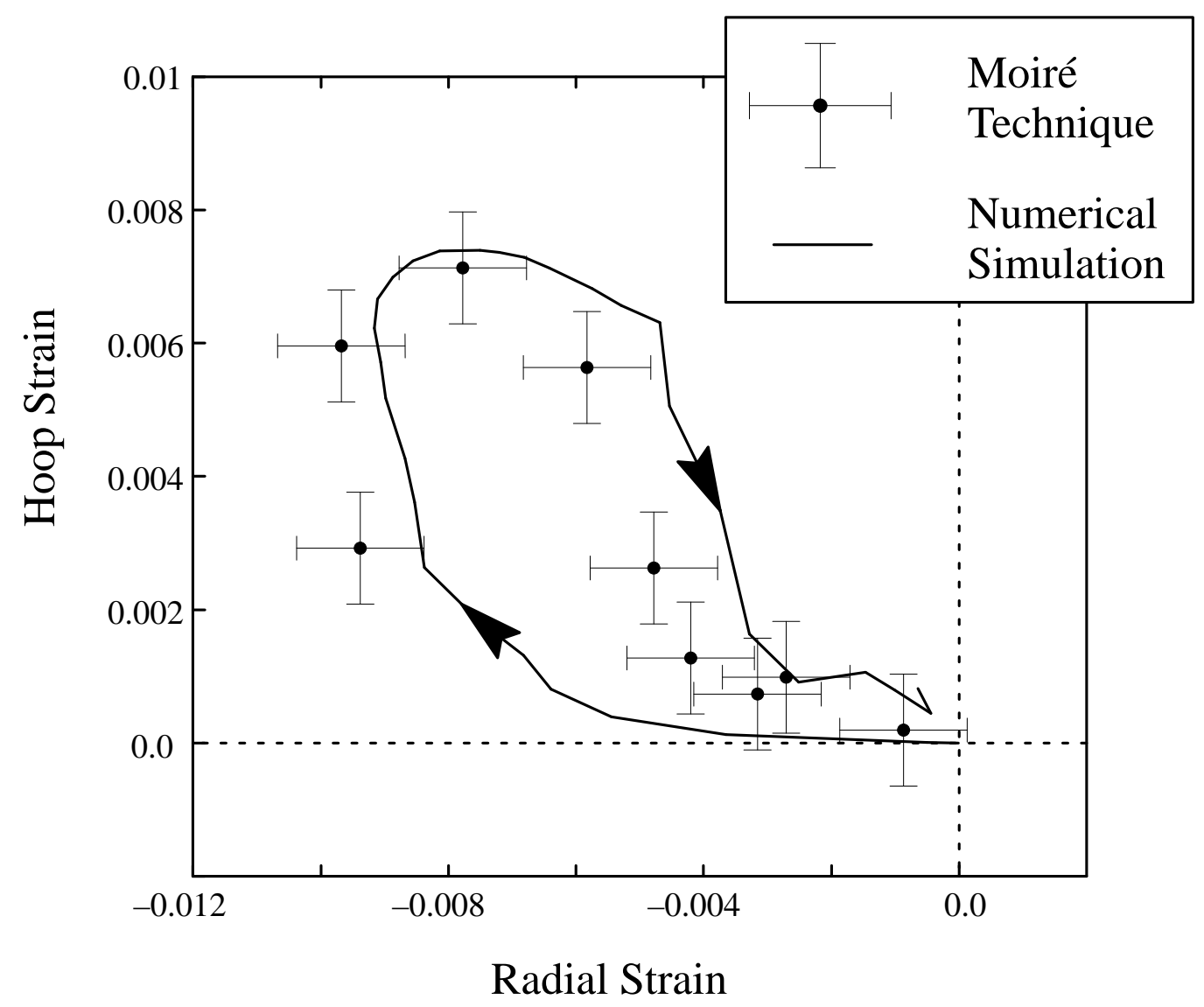

Fig. 7. Typical example of strain evolution given by a Moiré technique (dots). A comparison (curve) is proposed with the model. 